\title{
Nursing Care of HIV-Positive Women: A Scoping Literature Review on the Emotional Challenges for Women Living with a Chronic Disease
}

\author{
Ben Farid Røjgaard Nielsen and Bente Martinsen
}

\begin{abstract}
Living with HIV seems to be more stressful for women than men. Women exhibit higher levels of depression, lower levels of well-being and lower quality of life than HIVinfected men do. The aim of the review is to provide a comprehensive gender specific knowledge about the emotional challenges HIV infected women face in their daily lives. Across all articles one theme that emerged was the challenges about the social environment after being diagnosed with HIV. Three identified show what challenges women with HIV are facing in relation to employment, in relation to other people and a fear of stigma. Another theme that emerged was the challenges of different coping mechanisms. There was an evolution in how an HIV diagnosis had affected women's lives and their ability to cope with their diagnoses. Three identified subthemes show what challenges women with HIV are facing in relation to aiming to improve quality of life after being diagnosed with HIV, a sharp distinction between HIV and AIDS and a religious and spiritually coping. Identifying the emotional challenges women living with HIV face in their daily lives may help nurses obtain a clearer understanding and greater knowledge of how to provide HIV-positive women with effective care that empower and support these women in managing their chronic disease. However to ensure that nurses have the proper tools for effective care for women living with HIV European studies are essentials in relation to what emotional challenges these women are facing in their daily lives.
\end{abstract}

Keywords: coping strategies; HIV; nursing care, literature review, stigma, scoping, women

\section{INTRODUCTION}

Within recent years, dramatic scientific progress in the field of antiretroviral therapy (ART) has led to a significant decrease in mortality and morbidity among HIV-positive people. This development has transformed HIV from a being an acute terminal illness to being a manageable chronic condition. According to estimates from WHO and UNAIDS, 35.3 million people were living with HIV on a global scale in 2012, while 2.3 million were newly infected with HIV [1]. On a global scale, women comprise 52 percent of the HIVpositive population in low- and middle-income countries. In sub-Saharan Africa, however, women constitute approximately $57 \%$ of the HIV-positive population [1]. Young women and girls are particularly susceptible to contract HIV, and the global HIV prevalence among girls and young women is double or greater than among males of the same age [1]. Women exhibit higher levels of depression, lower levels of well-being and lower quality of life than men living with HIV [2]. Most of the existing studies deal with reproductive health, which is indeed an important area in the life of women living with HIV. However, this does not represent the totality of the female experience of living with HIV. In providing effective, professional care for HIVpositive women, nurses need to have a solid knowledge of the emotional challenges that women living with HIV face.

$$
\text { II. AIM }
$$

The focus is on the nurses' role in the ambulatory healthcare and in the setting of the home. Since HIV is a chronic disease, the aim is not to cure the disease, but to provide an effective chronic disease management. Apart from monitoring the patient's medication adherence, the nurse should also seek to empower and support people in living with HIV [3]. Since psychological problems and mood disorders are common in patients with HIV, nurses also have to intervene in relation to a variety of mental health issues. The aim of the review at hand is to provide a comprehensive gender specific knowledge about the emotional challenges HIV infected women face in their daily lives in order to improve the knowledge and tools available to nurses and other health care workers providing care for women living with HIV.

\section{METHODS}

The methodological approach employed in this study follows Arksey and O'Malley's guidelines for making a "scoping review" [4]. We followed five steps (table 1) in the scoping review and repeated as necessary to ensure to fully cover and investigate the existing body of literature. A qualitative content analysis [5] identifies gaps in the evidence-based studies as well as summarizing and disseminating findings.

\section{A. Scoping review}

A scoping review aims to map the key concepts underpinning a research area, providing a clear overview of the main sources of evidence available [5]. Unlike a systematic review the scoping study does not aim to synthesize evidence or to aggregate findings from different studies. Whilst a scoping review needs, an analytic framework in order to present a narrative account of existing literature there is no attempt made to present a view regarding the weight of evidence in relation to particular interventions. The reason is that the scoping study does not seek to assess quality of evidence and cannot determine where particular studies provide generalizable findings [5]. A key strength of 
the scoping study is that it can provide a rigorous and transparent method for mapping areas of research and makes it possible to identify the gaps in the evidence base.

Table 1. The five steps guiding the process of conducting our scoping study.

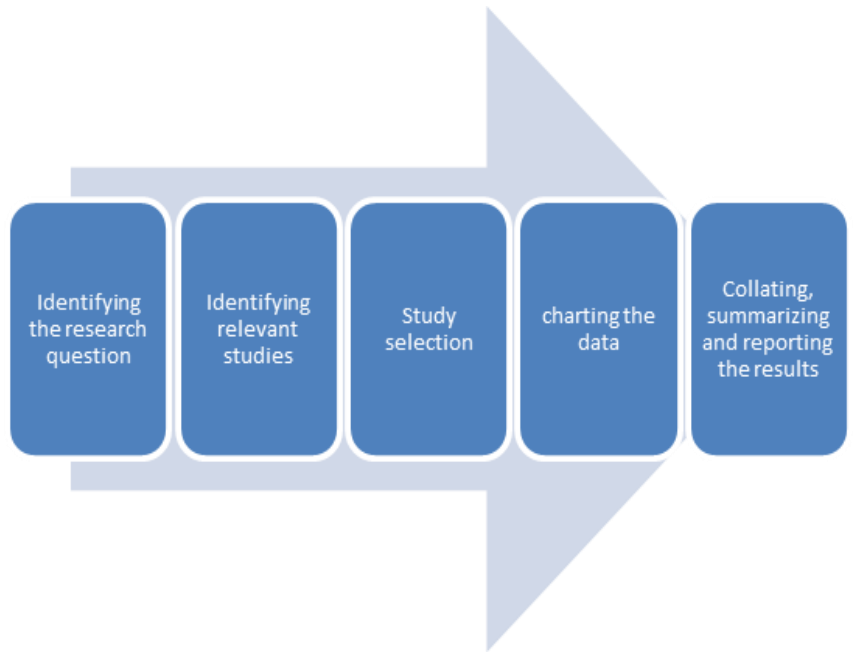

Step 1: Identifying the research question

The research question was: What knowledge does the existing literature offer on what emotional challenges HIVinfected women face in their daily lives?

Step 2 and 3: Identifying relevant studies and study selection

The point of scoping the field is to be as comprehensive as possible in identifying primary studies and reviews suitable for answering the review question. To achieve this a devised strategy that involved searching for research evidence articles via electronic databases and relevant organizations was made. A systematic search was performed in June 2014 using three electronic databases: PubMed, ProQuest Nursing and Allied Health Source. Because of the continuous development of new and improved medical treatment for HIV and its possible influence on the life conditions for women living with HIV, only studies on HIV infected women published between January 2009 and June 2014 were including. Only primary studies among adult biological women $(18+)$ were included. The complete list of article titles was scanned by two reviewers and assign a value of 'exclude', 'include' or 'maybe' to each reference. A total of ten articles were included in the scoping review.

Table 2. Flow chart of the retrieval of the studies (See Appendix I).

\section{Step 4: Charting the data}

In this stage, key items were charting of information and obtained from the primary research. The articles included in this review are based on both qualitative and qualitative methods. The analysis began by collecting general information from each of the ten articles including author(s), title and year of the study \& aim, methods, number of participants, age, country of investigation and ethical considerations (table 3).
Table 3. Reference, aim of study, methods, number and age of women included in the study, country and ethical considerations (See Appendix II)

We then proceeded to a qualitative analysis of the manifest content inspired by Graneheim \& Lundman [5]. An analysis of what the articles talks about deals with the content aspect and describe the visible obvious component referring as the manifest content. When reading the article the constellation of words or sentences relating to the same central meaning were condensed keeping its meaning and significance. The condensed meaning units were assigned to each code describing the contents of each unit. Codes that had common features were grouped into categories that reflect the core message. Each category refers mainly to a descriptive level of content and can thus be seen as an expression of the manifest content of the text. Each of the three identified categories contains a number of sub-categories.

\section{RESULTS}

The ten studies were conducted in six different countries, all outside Europe. The content analysis of the information provided by the studies including in the review leads to two thematic categories (table 3 ) or emotional challenges women living with HIV are facing:

- Challenges in the daily life about the social environment including challenges in relation to work, challenges in relation to other people and a fear of stigma.

- Challenges of coping; aiming to improve quality of life after being diagnosed with HIV, a distinction between HIV and AIDS and religious and spiritually coping.

Category 1: Challenges in the daily life about the social environment

Across all articles, one theme that emerged was the challenges about the social environment after being diagnosed with HIV. Three identified subthemes were closely related to each other and show what challenges women with HIV are facing in relation to work, in relation to other people and a fear of stigma

\section{1) Challenges in relation to work}

Women reported having difficulties in being accepted in the workplace and being afraid of losing their job: "I have been leaving my resumes for a long time, I believe the last job I had, the manager saw my documents in the drawer and, since this day, she has been treating me differently" [6]. Other women reported identity challenges when they could not work anymore: "I tried very hard to continue to work even though the doctor told me I shouldn't work. I kept trying to go back and even when I couldn't wear shoes because I had neuropathy so bad." [7].

Other women reports concern about their business if people found out they were HIV positive: "They will start talking. Like. Now I sell vegetables, they will say to others, 'How can you buy vegetables from her, she has AIDS; she 
has put the blood in the vegetables." [8].

2) Challenges in relation to other people

Some women described being labeled as promiscuous: "There are those who hate you who see you as a prostitute." [8]. Other women isolate themselves from other social situation and avoid situations where they make their health condition. They had a feeling of guilt for having caught a disease with sexual connotation [6]. Other women found themselves being labeled as having being infected because of ad deviant behavior such as infidelity or prostitution [8].

Women living with HIV seem to be concerned of overburdening their family, why some women did not disclosure their HIV status to any family members. Several women reported that they have been abandoned by friends or rejected by family members: "We used to get a number of our relatives coming to visit us, but now nobody wants to know how we are carrying on our life." [9]. Another woman explained about the discrimination from her sister: "Yes I told her, when I told her, she discriminated (against) me and gave me my plates, sheets, blankets, and my own room." [8].

In relation to women who were already in a relationship with a partner raised several challenges. For some there were feelings of anger and confusion toward their partner, primarily because that person was the most likely source of their HIV infection [9]. For others disclosing the HIV status to a partner was complicated because the women then were forced to reveal information that could cause tension in the relationships such as previous sexual behaviors, drug use or affairs.

\section{Fear of stigma}

Fear of stigma was found to be significantly and negatively associated with quality of life in all including studies. Some women identified HIV related stigma as some of the most difficult things they had experienced since being diagnosed with HIV [10]. Fear of stigma influenced on women's medication taking behavior, especially in public. Sometimes the women could not take their medication (ART) at home because members of the family had not been informed about their diagnosis. A study concludes that depression is prevalent among women infected with HIV and causes inadequate medication adherence [11].

\section{Category 2: Challenges of coping with HIV}

Across all articles, one theme that emerged was the challenges of different coping mechanisms. There was an evolution in how an HIV diagnosis had affected women's lives and their ability to cope with their diagnoses. Three identified subthemes were closely related to each other and show what challenges women with HIV are facing in relation to aiming to improve quality of life after being diagnosed with HIV, a sharp distinction between HIV and AIDS and a religious and spiritually coping.

\section{IMPROVING QUALITY OF LIFE UPON BEING DIAGNOSED WITH HIV}

Even there were women who continued to struggle with their HIV diagnosis, there were several women reporting that since being diagnosed with HIV they felt physically stronger, worked harder and had gained inner strength that allowed them to navigate in life's challenges [6,9] The HIV diagnose caused the women to change their habits, aiming to improve quality of life.

\section{A. The distinction between HIV and AIDS}

One study concludes that to cope with being HIV positive women make a sharp distinction between the meaning of HIV and AIDS with AIDS being marked as a state of hopelessness [12]. The women identified AIDS as a socially stigmatizing state in which friends and family could desert you though they had stood by you when you just had HIV. HIV positive women displace death and stigma away from an HIV diagnosis to an AIDS diagnosis creating a sense of normalcy for themselves [12]. A distinction between HIV and AID serves to distance the women emotionally away from a sense of physical, social and emotional threat why the author suggest the need to broaden the understanding of how illness meanings function for individuals patients to better support them in adhering to ART, in engaging in care and in living fully [12].

\section{B. Religion and spirituality as coping strategies}

The spiritual comfort acquired through religion plays a significant role for women coping with HIV in the daily life. Religion upon faith allowed the women to cope with being HIV positive and its impact on their daily life. A 35 years old widow with two children on ART for 3 months said: "When I feel sad I talk to Allah and accept my fate.” [13].

\section{CONCLUSION}

The impact of what emotional challenges women living with HIV are facing requires further research. However, by presenting the recent data from included studies in an accessible and summarized format, this review may contribute to nurse's understanding and knowledge when caring for women living with HIV in order to empower and support these women's chronic disease management. According to studies included in this review, women with HIV are facing emotional challenges in the daily life in relation to the social environment, including challenges in relation to work, challenges in relation to other people and a fear of stigma. Another emotional challenge women living with HIV are facing in their daily life is challenges of coping with the disease, including aiming to improve quality of live after being diagnosed with HIV, a distinction between HIV and AIDS and religious and spiritually coping.

\section{REFERENCES}

[1] HIV/AIDS: How many people are living with HIV? (2013, October 30). Retrieved may 5, 2014: http://www.who.int/features/qa/71/en/ 
[2] M. R. Loutfy, L. Sherr, U. Sonneberg-Schwan, S. L. Walmsley, M. Johnson and A. d'Arminio Monforte,"Caring for women living with HIV: gaps in the evidence," in Journal of the International AIDSsociety, vol. 16, pp.18509. 2013.

[3] Family Health International (FHI), "Nursing care of patients with HIV/AIDS: Facilitator's guide".2007.This document was funded by the US

Agency for International Development (USAID) through FHI's Implementing AIDS Prevention and Care (IMPACT) project, Cooperative Agreement HRN-A-00-97-00017-00.Retrived may 5 2014:

http://www.fhi360.org/sites/default/files/media/documents/Nursing\%20Car e\%20of\%20Patients\%20with\%20HIV-AIDS\%20$\% 20$ Facilitator\%20Guide.pdf

[4] H. Arksey and L. O'Malley, “Scoping studies: towards a methodological framework," in International Journal of Social Research Methodology, vol. 8(1), pp. 19-32, 2005.

[5] U. H. Graneheim, and B. Lundman, "Qualitative content analysis in nursing research: concepts, procedures and measures to achieve trustworthiness," in Nurse Education Today, vol. 24 (2) pp. 105-112, February 2004.

[6] L. M. Silva, M. A. Moura, and M. L. Pereira, "The daily life of women after HIV/AIDS infection: guidelines for nursing care," in Texto \& Contexto - Enfermagem, vol. 22. Vol 22, no2. pp. 335-342, Florianopolis Apr/June 1013.

[7] J. Peterson, "The challenge of seeking and receiving support for women living with HIV," in Health Communication, vol. 25(5), pp. 470-479, July 2010

[8] P. Kako and R. Dubrosky, "You comfort yourself and believe in yourself- Exploring lived experienced of Stigma in HIV positive Kenyan women," in Mental Health Nursing, vol. 34 (3) pp. 150-157, March 2013.

[9] A. Medley, C. E. Kennedy, S. Lunyolo, and M. D. Sweat, "Disclosure outcomes, coping strategies and life changes among women living with HIV in Uganda," in Qualitative Health Research, vol. 19, pp. 1744-1754, December 2009.

[10] M. Vyavaharkar, L. Moneyham, C. Murdaugh and A. Tavakoli, "Factors Associated with quality of life among rural women with HIV disease," in AIDS Behavior, vol. 16 (2), pp. 295-303, February 2012.

[11] A. M. Badahdah, and D. E. Pedersen, "I want to stand on my own legs. A qualitative study of antiretroviral therapy adherence among HIV positive women in Egypt," in AIDS Care, vol. 23(6), pp. 700-704, June 2011
[12] E.Lewis "Depression in HIV positive Women in Gaborone, Botswana," in Health Care for Women International, 33(4), pp.375386.April 2012

[13] A. Scott, "Illness meanings of AIDS among women with HIV. Merging Immunology and life experience," in Qualitative Health Research, vol. 19(4), pp. 454-465, April 2009.

\section{AUTHORS' PROFILE}

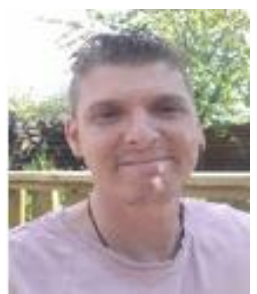

Ben Nielsen is a Lecturer at the School of Nursing in Metropolitan University College, Denmark. He is a trained nurse and is Master of Science (MSc) in nursing from Aarhus University, Denmark in 2007. Nielsen research is concerned with nursing and intercultural communication.

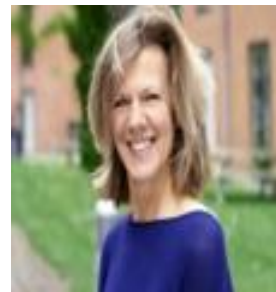

Bente Martinsen is an Associate Professor at Aarhus University, Denmark. Her research is concerned with peoples' experiences of physical dependency with a special focus on the phenomenon of assisted feeding. 
Appendix I

Table 2. Flow chart of the retrieval of the studies.

Search terms:
women"[MeSH Terms]
OR "women"[All
Fields]) AND
("hiv"[MeSH Terms] OR
"HIV"[All Fields]) AND
("mental health"[MeSH
Terms] OR
("mental"[All Fields]
AND "health"[All
Fields]) OR "mental
health"[All Fields] OR
("emotional"[All Fields]
AND "health"[All
Fields]) OR "emotional
health"[All Fields]))
AND
("2009/06/27"[PDat] :
"2014/06/25"[PDat])
Limits: English
language, years 2009-
2014,
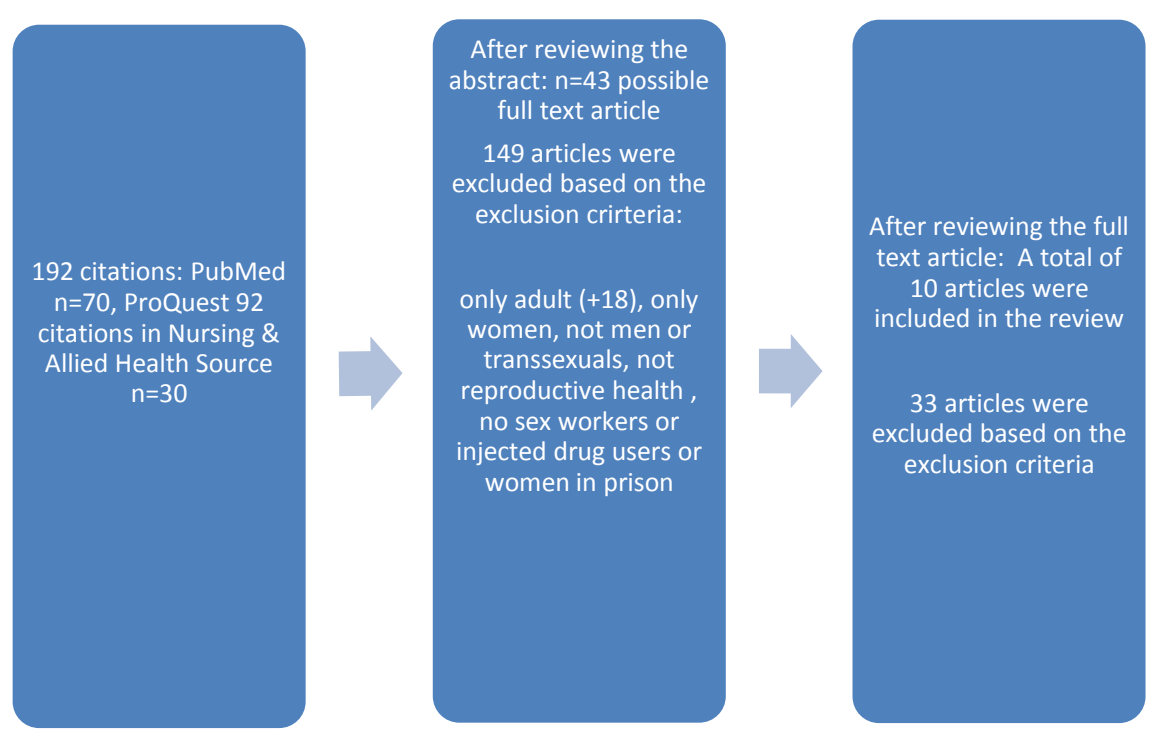


\section{Appendix II}

Table 3. Reference, aim of study, methods, number and age of women included in the study, country and ethical considerations.

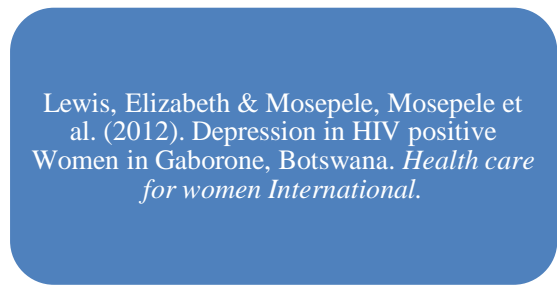

- Aim: Measuring prevalence of depression and suicide among HIVpositive women in Botswana.

- Methods: Cross-sectional study. 62 women aged 23-53.

- Ethics: Written informed consent was obtained from the women after the study has been explained to them

Peterson, Jenifer. (2010). The challenge of seeking and receiving support for women living with HIV. Health Communication.

Kako, Peninnah \& Dubrosky, Rebekah.

(2013). "You comfort yourself and believe in yourself." Exploring lived experienced of Stigma in HIV positive Kenyan women. Mental Health Nursing.

Vyavaharkar, Medha \& Moneyham, Linda et al. (2012). Factors Associated with quality of life among rural women with HIV disease. AIDS Behavior.

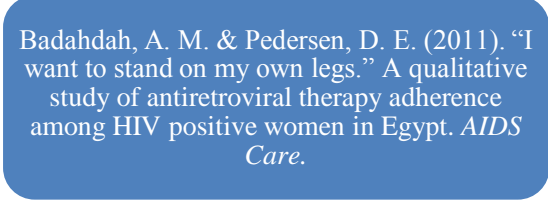

Reis, Renata K. \& Santos, C. B. et al. (2012). Quality of life among Brazilian women living with HIV/AIDS. AIDS Care

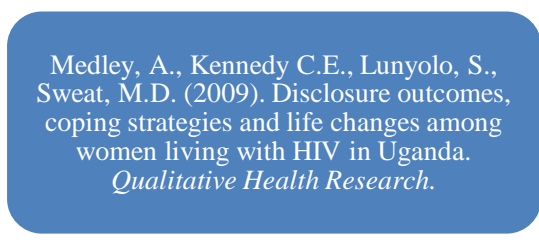

Hickman, Enith E., Glass, Carol R., Arnkoff, Diane B. \& Fallot, Roger D. (2013). Religious coping, stigma and psychological functioning among HIV positive African American women. Mental Health, Religion \& Culture.

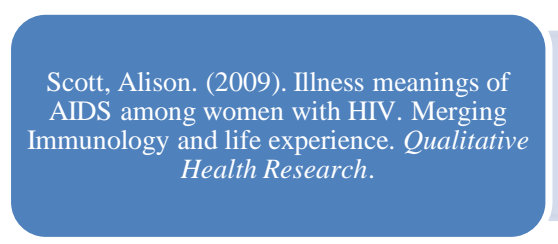

- Aim: Exploring the social challenges among HIV-positive women from Midwestern cities in the US.

- Methods: A qualitative grounded theory approach. 45 women aged 1945.

- Ethics: The women were ask to sign an informed consent document

- Aim: Exploring experiences of stigma among HIV-positive women in Kenya.

- Methods: Longitudinal qualitative narrative methodology 54 women, average age 37.

- Ethics: Women were informed of the voluntary nature of the study. Consent and written statement.

- Aim: Examining physical, psychological and social factors associated with quality of life among rural women with HIV in the Southeastern US.

- Methods: Cross-sectional study. 399 women aged 18+

- Ethics: Participants were provided with written information about the study.

- Aim: Examining the factors that influence women's adherence to HIV medications in the Arab world.

- Methods: Qualitative study. Face to face interviews with 27 women aged 22-52 from Egypt.

- Ethics: The women were informed that their participation was entirely voluntary

- Aim: Assessing the quality of life among HIV-positive women in Sao Paolo, Brazil.

- Methods: Cross-sectional study. 106 women ages 18+.

- Ethics: No information available on the ethical aspects

- Aim: To explore links between HIV disclosure and coping strategies among women living with HIV.

- Methods: Qualitative in- depth interviews30 women, median age 28 from Kampala, Uganda.

- Ethics: Oral and written informed consent was obtained

- Aim: To examine the role of religious coping in psychological distress and adjustment among women living with HIV in Washington DC, USA.

- Methods: Cross-sectional and longitudinal study of 141 women aged 20-64.

- Ethics: Informed consent

- Aim: To explore meaning surrounding the concept of AIDS among women with HIV.

- Methods: Multiple qualitative methods, 10 women aged 18-24 from New Orleans, Louisiana, USA.

- Ethics: Recently diagnosed or physically vulnerable women were not approached 
Silva, L. M., Moura, M. A. \& Pereira, M.

L. (2013). The daily life of women after

HIV/AIDS infection: guidelines for

nursing care. Texto \& Contexto Enfermagem,
- Aim: To understand the daily lives of women after HIV/AIDS infection as a support to guide nursing care in relation to women's healthcare,

- Methods: Qualitative and descriptive research based on indiviudal interviews. 39 women aged 18+ from Fortaleza, the state of Ceará, Brazil.

- Ethics: Informed consent forms were used.

In total:

1 study from Botswana, 1 from Egypt, 1

from Kenya, 1 from Uganda, 2 studies

from Brazil and 4 studies from the US. 